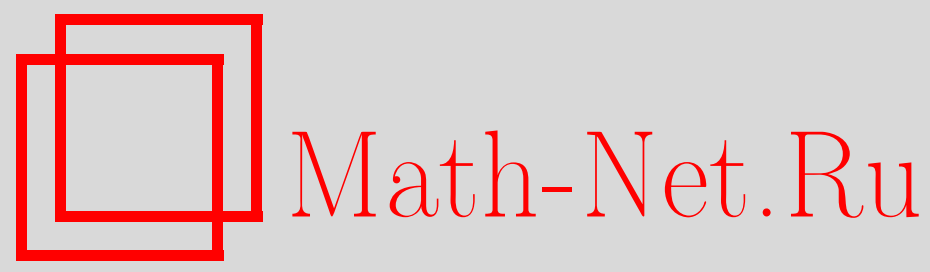

Т. Л. Мелехина, Конструкция канонических координат на орбитах коприсоединенного представления тензорных расширений групп Ли, Матем. заметки, 1998, том 64, выпуск 2, 318-320

DOI: https://doi.org/10.4213/mzm1402

Использование Общероссийского математического портала Math-Net.Ru подразумевает, что вы прочитали и согласны с пользовательским соглашением http://www.mathnet.ru/rus/agreement

Параметры загрузки:

IP : 54.210 .77 .194

26 апреля 2023 г., $12: 17: 56$

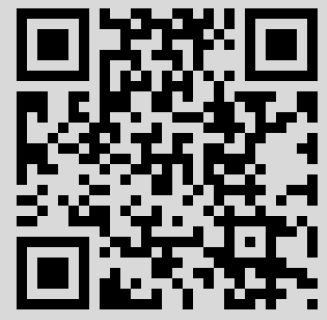




\section{КОНСТРУКЦИЯ КАНОНИЧЕСКИХ КООРДИНАТ НА ОРБИТАХ КОПРИСОЕДИНЕННОГО ПРЕДСТАВЛЕНИЯ ТЕНЗОРНЫХ РАСШИРЕНИЙ ГРУПП ЛИ}

\section{Т. Л. Мелехина}

В настоящее время большой цикл работ посвящен построению канонических координат на симплектических многообразиях. Важный класс симплектических многообразий составляют орбиты коприсоединенного представления групп Ли, на которых реализуются многие важные гамильтоновы системы. В работе [1] для нахождения глобальных симплектических координат на орбитах максимальной размерности коприсоединенного представления $\mathrm{Ad}^{*}$ нильпотентных групп Ли $\mathscr{T}$ использовались цепочки идеалов $G_{1} \subset G_{2} \subset \cdots \subset G_{n}=G$ таких, что $\operatorname{dim} G_{i} / G_{i-1}=1$, и инварианты представления $\mathrm{Ad}^{*}$ групп Ли $\mathscr{T}_{i}$, отвечающих идеалам $G_{i}, i=1, \ldots, \operatorname{dim} G$, поднимались на $G^{*}$ с помощью естественной проекции $p_{i}: G^{*} \rightarrow G_{i}^{*}$, где $\mathscr{T}$ - группа Ли, отвечающая алгебре Ли $G$. В итоге получались требуемые симплектические координаты. Канонические координаты на орбитах общего положения коприсоединенного представления $\mathrm{Ad}^{*}$ группы Ли верхнетреугольных невырожденных матриц четвертого порядка найдены в [2]. Авторы [3] предложили общий алгоритм построения канонических координат на орбитах, обладающих поляризацией.

Пусть $A$ - коммутативное кольцо, $G$ - алгебра Ли. Тогда их тензорное произведение $G \otimes A$ является алгеброй Ли относительно операции коммутирования $[g \otimes a, h \otimes b]=[g, h] \otimes a b$, где $g, h \in G, a, b \in A$. В случае, когда кольцо $A$ является алгеброй с двойственностью Пуанкаре, в работе [4] В.В. Трофимов предложил алгоритм $(t)$, позволяющий продолжать функции с пространства $G^{*}$, сопряженного к алгебре Ли $G$, на пространство $(G \otimes A)^{*}$, сопряженное к алгебре Ли $G \otimes A$ (см. также [5] и [6]). В работе [7] показано, что эта конструкция может быть использована для построения канонических координат на орбитах коприсоединенного представления $\mathrm{Ad}^{*}$ группы Ли, отвечающей алгебре Ли $G \otimes\left(\mathbb{R}[x] /\left(x^{2}\right)\right)$, где $G$ - произвольная алгебра Ли, на орбитах которой такая система координат явно указана. В работе [8] показано, что это утверждение остается справедливьг для кольца $\mathbb{R}[x] /\left(x^{3}\right)$.

В настоящей работе доказано, что алгоритм $(t)$ Трофимова, примененньй к функциям, задающим канонические координаты на орбитах коприсоединенного представления $\mathrm{Ad}^{*}$ группы Ли $\mathscr{T}$, дает канонические координаты на орбитах коприсоединенного представления $\mathrm{Ad}^{*}$ группы Ли $\mathscr{T} \otimes K$, отвечающей алгебре Ли $G \otimes K$, где $G$ - алгебра Ли группы Ли $\mathscr{T}$ и $K=\mathbb{R}[x] /\left(x^{n}\right)$. Для этого кольца описано действие алгоритма $(t)$ в явном виде, что позволило продолжить его на более широкий класс функций по сравнению с работами [4], [5].

Пусть $K=\mathbb{R}[x] /\left(x^{n}\right)$, а $G$ - произвольная алгебра Ли. Предположим, что $e_{1}, \ldots, e_{r}-$ базис алгебры Ли $G, r=\operatorname{dim} G$. Тогда

$$
e_{1}, \ldots, e_{r}, e_{1} \otimes \varepsilon, \ldots, e_{r} \otimes \varepsilon, \ldots, e_{1} \otimes \varepsilon^{n-1}, \ldots, e_{r} \otimes \varepsilon^{n-1}
$$

- базис алгебры Ли $G \otimes K$, где $\varepsilon=\pi(x)$ и $\pi: \mathbb{R}[x] \rightarrow K$ - каноническая проекция. Координаты в пространстве $(G \otimes K)^{*}$ в базисе, дуальном к базису пространства $G \otimes K$, обозначим через $x_{i_{1}}(0), x_{i_{2}}(1), \ldots, x_{i_{n}}(n-1)$, причем $x_{i_{1}}(0)$ относится к базису $e^{i_{1}} \in G^{*}, x_{i_{2}}(1)$ - к базису $e^{i_{2}} \otimes \varepsilon \in(G \otimes \varepsilon)^{*}$, и т.д. $x_{i_{n}}(n-1)-$ к базису $e^{i_{n}} \otimes \varepsilon^{n-1} \in\left(G \otimes \varepsilon^{n-1}\right)^{*}$, где $e^{1}, \ldots, e^{n}$ - дуальный базис в $G^{*}$, т.е. $e^{i}\left(e_{j}\right)=\delta_{j}^{i}$. Пусть $C_{i j}^{k}$ - структурный тензор алгебры Ли $G$ в базисе $e_{1}, \ldots, e_{n}$ и

$$
z_{i}=x_{i}(0) \varepsilon^{n-1}+x_{i}(1) \varepsilon^{n-2}+\cdots+x_{i}(n-1) .
$$

Предположим, что функция $F(z)$ допускает разложение в ряд Тейлора. Тогда можно записать равенство

при условии, что $\varepsilon^{n}=0$.

$$
F(z)=F^{(0)}(z)+F^{(1)}(z) \varepsilon+\cdots+F^{(n-1)}(z) \varepsilon^{n-1}
$$

Алгоритм $(t)$ Трофимова переводит функцию $F(z)$, определенную на пространстве $G^{*}$, в набор функций $F^{(0)}, \ldots, F^{(n-1)}$ (см., например, [6]). 
ЛЕмма 1. Функиии $F^{(m)}, m=0, \ldots, n-1$, имеют следующий вид:

$$
\begin{aligned}
F^{(m)}(z)=\frac{1}{m !} & \frac{\partial^{m} F\left(x_{i}(m)\right)}{\partial x_{i_{1}} \cdots \partial x_{i_{m}}} \prod_{i=i_{1}}^{i_{m}} x_{i}(m-1) \\
& +\frac{1}{(m-2) !} \frac{\partial^{m-1} F\left(x_{i}(m)\right)}{\partial x_{i_{1}} \cdots \partial x_{i_{m-1}}} \prod_{i=i_{1}}^{i_{m-2}} x_{i}(m-1) x_{i_{m-1}}(m-2) \\
& +\frac{1}{(m-4) !} \frac{\partial^{m-2} F\left(x_{i}(m)\right)}{\partial x_{i_{1}} \cdots \partial x_{i_{m-2}}}\left(\prod_{i=i_{1}}^{i_{m-3}} x_{i}(m-1) x_{i_{m-2}}(m-3)\right. \\
& \left.+\frac{1}{(m-2) !} \prod_{i=i_{1}}^{i_{m-4}} x_{i}(m-1) x_{i_{m-3}}(m-2) x_{i_{m-2}}(m-2)\right) \\
& +\cdots+\frac{\partial F\left(x_{i}(m)\right)}{\partial x_{i}} x_{i}(0) .
\end{aligned}
$$

Обобщим алгоритм $(t)$. Функции, приведенные в лемме 1 , построены в предположении, что $F$ разложима в ряд Тейлора. Имея явные выражения для $F^{(m)}$, мы можем определить $F^{(m)}$ для любых гладких функций $F$, заданных напространстве $G^{*}$. По определению положим, что алгоритм $(t)$ переводит функцию $F$ в набор $F^{(0)}, \ldots, F^{(m-1)}$ функций, описанных в лемме 1.

Имеет место основная теорема.

Теорема 1. Пусть функиии $p_{1}, \ldots, p_{s}, q_{1}, \ldots, q_{s}$, определеннъе на пространстве $G^{*}$, дают канонические координаты на всех орбитах общего положсения коприсоединенного представления $\mathrm{Ad}^{*}$ әруппь Ли, отвечающей алгебре Ли $G$, т.е.

$$
\left\{p_{i}, p_{j}\right\}=\left\{q_{i}, q_{j}\right\}=0, \quad\left\{p_{i}, q_{j}\right\}=\delta_{i j}
$$

Тогда функиии

$$
\begin{gathered}
P_{1}=p_{1}^{(0)}, \quad \ldots, \quad P_{s}=p_{s}^{(0)}, \quad P_{s+1}=p_{1}^{(1)}, \ldots, \\
P_{2 s}=p_{s}^{(1)}, \quad \ldots, \quad P_{s(n-1)+1}=p_{1}^{(n-1)}, \quad \ldots, \quad P_{s n}=p_{s}^{(n-1)}, \\
Q_{1}=q_{1}^{(n-1)}, \quad \ldots, \quad Q_{s}=q_{s}^{(n-1)}, \quad \ldots, \quad Q_{s(n-1)+1}=q_{1}^{(0)}, \quad \ldots, \quad Q_{s n}=q_{s}^{(0)}
\end{gathered}
$$

на пространстве $(G \otimes K)^{*}$ дают канонические координаты на всех орбитах общего положения коприсоединенного представления $\mathrm{Ad}^{*}$ группь Ли, отвечающей алгебре Ли $G \otimes K$, m.e.

$$
\left\{P_{i}, P_{j}\right\}=\left\{Q_{i}, Q_{j}\right\}=0, \quad\left\{P_{i}, Q_{j}\right\}=\delta_{i j}
$$

Отметим в качестве следствия из теоремы 1 следующий результат.

Tеорема 2. Пусть $G-$ неразложимая вещественная алгебра $Л и, \operatorname{dim} G=3,4,5, G \not$ sо(3), либо $G$ - неразложимая вещественная нильпотентная аләебра Ли, $\operatorname{dim} G=6$. Используя алгоритм $(t)$, мохно в явном виде построить глобальную систему канонических координат на всех орбитах общего положения коприсоединенного представления Ad $^{*}$ группь Ли, отвечающей алгебре Ли $\left(\left(\left(G \otimes K_{1}\right) \otimes K_{2}\right) \otimes \cdots \otimes K_{s}\right)$, где $K_{i}=\mathbb{R}[x] /\left(x^{n_{i}}\right)$.

Явные выражения для функций, приведенных в лемме 1 , получаются прямым вычислением с использованием формулы Тейлора. Для функций $F^{(m)}(w)$, где $w$-полный набор координат $x_{i}(j)$, 
$i=1, \ldots, r, j=0, \ldots, n-1$, выполняется соотношение

$$
\begin{aligned}
& \frac{\partial F^{(m)}(w)}{\partial x_{i}(0)}\left(\frac{1}{m !} \prod_{i=i_{1}}^{i_{m}} x_{i}(m-1) \frac{\partial^{(m)}}{\partial x_{i_{1}} \cdots \partial x_{i_{m}}}\left(C_{i j}^{k} w_{k} \frac{\partial F}{\partial w_{j}}\right)+\cdots\right) \\
& +\frac{\partial F^{(m)}(w)}{\partial x_{i}(1)}\left(\frac{1}{(m-1) !} \prod_{i=i_{1}}^{i_{m-1}} x_{i}(m-2) \frac{\partial^{(m-1)}}{\partial x_{i_{1}} \cdots \partial x_{i_{m-1}}}\left(C_{i j}^{k} w_{k} \frac{\partial F}{\partial w_{j}}\right)+\cdots\right)+\cdots \\
& +\frac{\partial F^{(m)}(w)}{\partial x_{i}(m-1)}\left(C_{i j}^{k} w_{m} \frac{\partial F}{\partial w_{j}}\right) \\
& =\left(\frac{1}{m !} C_{i j}^{k} w_{k} \frac{\partial}{\partial x_{i}(0)}\left(\frac{\partial F}{\partial x_{i}} x_{i}(0)\right) \frac{\partial^{(m)}}{\partial x_{i_{1}} \cdots \partial x_{i_{m}}}\left(\frac{\partial F}{\partial w_{j}} \prod_{i=i_{1}}^{i_{m}} x_{i}(m-1)\right)+\cdots\right) \\
& +\left(\frac{1}{(m-1) !} C_{i j}^{k} w_{k} \frac{\partial}{\partial x_{i}(1)}\left(\frac{\partial^{2} F}{\partial x_{i} \partial x_{j}} x_{i}(m-1) x_{j}(1)\right)\right. \\
& \left.\quad \times \frac{\partial^{(m-1)}}{\partial x_{i_{1}} \cdots \partial x_{i_{m-1}}}\left(\frac{\partial F}{\partial w_{j}} \prod_{i=i_{1}}^{i_{m-1}} x_{i}(m-1)\right)+\cdots\right)+\cdots \\
& +\left(C _ { i j } ^ { k } w _ { k } \frac { \partial } { \partial x _ { i } ( m - 1 ) } \left(\frac{\partial^{(m)} F}{\partial x_{i_{1}} \cdots \partial x_{i_{m}}} \prod_{i=i_{1}}^{i_{m}} x_{i}(m-1)\right.\right. \\
& \left.\left.+\frac{1}{(m-2) !} \frac{\partial^{(m-1)}}{\partial x_{i_{1}} \cdots \partial x_{i_{m-1}}} \prod_{i=i_{1}}^{i_{m-2}} x_{i}(m-1) x_{i_{m-1}}(m-2)+\cdots\right) \frac{\partial F}{\partial w_{j}}\right) \\
& =C_{i j}^{k} w_{k} \frac{\partial}{\partial w_{p}}\left(w_{p} \frac{\partial F^{(n)}}{\partial w_{i}} \frac{\partial F^{(n)}}{\partial w_{j}}\right) .
\end{aligned}
$$

Поскольку скобка Пуассона $\{f, h\}$ произвольных функций $f, h$, определенных на пространстве $(G \otimes K)^{*}$, имеет вид

$$
\{f, h\}=C_{i j}^{k} w_{k} \frac{\partial f}{\partial w_{i}} \frac{\partial h}{\partial w_{j}},
$$

теорема 1 следует из равенства (1), примененного к функциям $P_{i}$ и $Q_{j}$.

\section{СПИСОК ЦИТИРОВАННОЙ ЛИТЕРАТУРЫ}

1. Vergne M. // Bull. Soc. Math. France. 1972. V. 100. P. 301-335. 2. Новак E. // Tp. ceминара по векторному и тензорному анализу. 1993. Вып. 25. Ч. 2. С. 4-22. 3. Kamalin А. А., Perelomov A. M.// Comm. Math. Phys. 1985. V. 97. Р. 553-568. 4. Трофимов В. В. // Изв. АН СССР. Сер. матем. 1983. Т. 47. №6. С. 1303-1331. 5. Браилов А. В. // Вестн. МГУ. Сер. 1. Матем., мех. 1983. №1. С. 47-51. 6. Трофимов В.В., Фоменко А. Т. Алгебра и геометрия интегрируемых гамильтоновых дифференциальных уравнений. М.: Факториал, 1995. 7. Трофимов В. В. // УМН. 1994. Т. 49. № 1. С. 229-230. 8. Мордашева Т. Л. // УМН. 1995. Т. 50. №6. C. $193-194$.

Московский государственный педагогический университет

Поступило им. В. И. Ленина 